\title{
Comparison of osteoporotic fracture risk in surgical and natural menopausal patients
}

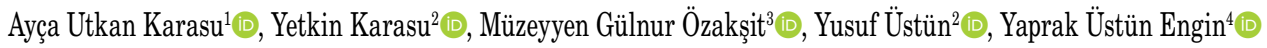 \\ ${ }^{1}$ Department of Physical Medicine and Rehabilitation, Gazi University School of Medicine, Ankara, Turkey \\ ${ }^{2}$ Department of Obstetrics and Gynecology, University of Health Sciences, Ankara Training and Research Hospital, Ankara, Turkey \\ ${ }^{3}$ Department of Obstetrics and Gynecology, University of Health Sciences, Ankara City Hospital, Ankara, Turkey \\ ${ }^{4}$ Department of Obstetrics and Gynecology, University of Health Sciences, Etlik Zübeyde Hanım Maternity and Women's Health Hospital Research Hospital, Ankara, Turkey
}

Received: January 12, 2020 Accepted: May 07, 2020 Published online: September 01, 2021

\begin{abstract}
Objectives: This study aims to compare the fracture risk calculated with Fracture Risk Assessment Tool (FRAX $\left.{ }^{\circledast}\right)$ in patients with natural and surgical menopause.

Patients and methods: Between April 2019 and July 2019, 285 postmenopausal patients (mean age 57.3 years; range, 40 to 78 years) who were admitted to the menopause clinic were enrolled in this prospective cross-sectional study. Of these, 220 were in natural menopause and 65 were in surgical menopause. Demographic data, medical history, and International Physical Activity Questionnaire scores were collected through face-to-face interviews with the patients. Femoral neck and lumbar vertebrae (L1-L4) T-scores were evaluated using dual-energy $\mathrm{X}$-ray absorptiometry. Fragility fracture risk was assessed using FRAX ${ }^{\circledR}$.

Results: The groups were similar in terms of age, body mass index, duration of menopause, smoking, alcohol use, and history of fracture $(\mathrm{p}>0.05)$. The risk of major osteoporotic fracture and hip fracture calculated without adding bone mineral density (BMD) was similar between groups $(\mathrm{p}=0.417$ and $\mathrm{p}=0.234)$. The risk of hip fracture calculated with the addition of BMD was higher in natural menopause patients $(\mathrm{p}=0.023)$. Lumbar vertebrae T-scores were similar between two groups regardless of age; femoral neck T-scores were higher in surgical menopause $(\mathrm{T}$-score $=-0.8)$ than natural menopause group $(\mathrm{T}$-score $=-1.25)$ aged under 60 years, whereas this difference disappeared after 60 years of age.

Conclusion: In our study, the fracture risk and the severity of osteoporosis were not different in surgical menopausal patients compared to the natural menopausal patients. Hip fracture risk calculated using BMD was lower in patients under 50 years of age in surgical menopausal patients. However, the fracture risks were similar in both groups after 50 years of age.
\end{abstract}

Keywords: Fracture risk assessment tool, menopause, osteoporosis, osteoporotic fractures.

Natural menopause is the complete cessation of menstrual periods without any surgical intervention or medical treatment. It is characterized by very low estrogen and high follicle-stimulating hormone levels, with depletion of ovarian follicles around the age of fifty years. Surgical menopause is defined as menopause after bilateral oophorectomy. ${ }^{[1]}$
During reproductive years, ovaries produce estradiol, testosterone, and androstenedione. After natural menopause, ovaries continue to produce androstenedione and testosterone for many years. These androgens are converted to estrone, the primary estrogen in the postmenopausal period in fat, muscle, and skin tissues. Premenopausal oophorectomy

\footnotetext{
Corresponding author: Ayça Utkan Karasu, MD. Gazi Üniversitesi Tip Fakültesi Fiziksel Tıp ve Rehabilitasyon Anabilim Dalı, 06560 Yenimahalle, Ankara, Türkiye. e-mail: aycautkan@gazi.edu.tr 
results in sudden reduction of these hormones. Serum androstenedione and testosterone plasma levels of females with surgical menopause have been shown to be significantly lower than in females with natural menopause. Estrogens and androgens inhibit bone resorption, and androgens increase bone formation. ${ }^{[2]}$

There are reports indicating that bone loss can reach up to $20 \% 18$ months after bilateral oophorectomy. Bilateral oophorectomy before the age of 45 years is a long-established risk factor for osteoporosis. There are studies indicating that oophorectomy during menopause may increase the risk of osteoporosis. In general, there is increasing evidence that bilateral oophorectomy may be associated with increased bone loss, higher osteoporosis rates, and fracture risk..$^{[1]}$

The primary goal of osteoporosis treatment is to reduce the risk of fragility fractures. It is critical to evaluate the risk of fracture in patients who need treatment. In 2008, in Sheffield, United Kingdom, the World Health Organization Collaboration Center introduced the Fracture Risk Assessment Tool $\left(\right.$ FRAX $\left.^{\circledR}\right)$, which is used to assess a person's 10-year risk of fracture. FRAX ${ }^{\circledast}$ uses eight clinical risk factors (previous fracture, hip fracture in parents, smoking and systemic glucocorticoid use, excessive alcohol consumption, body mass index [BMI], rheumatoid arthritis, and history of secondary osteoporosis), age, and gender for risk assessment. The measurement of bone mineral density (BMD) of the femoral neck is optional. ${ }^{[3]}$

Fracture Risk Assessment Tool is a computerbased algorithm (http://www.shef.ac.uk/FRAX) that computes a 10-year probability of major osteoporotic fracture (hip, clinical spine, humerus, or wrist fracture) and the probability of hip fracture for 10 years. Fracture Risk Assessment Tool models have been made available for 64 countries and in 31 languages covering more than $80 \%$ of the world population. ${ }^{[4]}$ There is a FRAX $^{\circledR}$ model that has been adapted to Turkey and available online (https://www.sheffield.ac.uk/FRAX/ charts/Chart_TE_ost_wom_bmd.pdf). ${ }^{[5]}$ In this study, we aimed to compare the fracture risk calculated with FRAX $^{\circledR}$ in patients with natural and surgical menopause.

\section{PATIENTS AND METHODS}

This prospective cross-sectional study was conducted between April 2019 and July 2019 in Zekai Tahir Burak Women's Health Training and Research Hospital, Physical Medicine and Rehabilitation Clinic.
A total of 285 postmenopausal patients mean age 57.3 years; range, 40 to 78 years) who were admitted to the menopause clinic for annual check-ups were enrolled in the study. Of these, 220 were in natural menopause and 65 were in surgical menopause. Patients were excluded if they had previously received pharmacological treatment for osteoporosis. The study protocol was approved by the Zekai Tahir Burak Women's Health Training and Research Hospital Ethics Committee (Approval No.: 44/2019). A written informed consent was obtained from each patient. The study was conducted in accordance with the principles of the Declaration of Helsinki.

Demographic data, medical history, and International Physical Activity Questionnaire (IPAQ) scores of the patients were collected through face-toface interviews with the patients. Femoral neck and lumbar vertebrae (L1-L4) T-scores were evaluated using dual energy X-ray absorptiometry (DXA). Fragility fracture risk was assessed using FRAX ${ }^{\circledR}$. The risk of major osteoporotic fractures was calculated in two ways: without including femoral neck T-scores (FRAX $^{\circledR}$ Major Osteoporotic [MO] and FRAX ${ }^{\circledR}$-Hip) and including femoral neck T-scores (FRAX ${ }^{\circledR} \mathrm{MO} / \mathrm{BMD}$ and FRAX ${ }^{\circledR}$ Hip/BMD). ${ }^{[6]}$ The IPAQ is a well-established questionnaire used to obtain comparable estimates of physical activity. The Turkish version of IPAQ was used for the physical activity assessment. ${ }^{[7]}$ The IPAQ questions the amount and type of physical activity per week. As a result of the IPAQ assessment, the scores of individuals are determined as metabolic equivalent (MET). A MET is a multiple of your estimated resting energy expenditure. One MET is what you spend when you are at rest. Therefore, 2 METs are twice what you spend at rest, walking is 3.3 METs, moderate physical activity is 4 METs, and vigorous physical activity is 8 METs. Natural menopause and surgical menopause groups were compared in terms of demographic and clinical characteristics, FRAX ${ }^{\circledast}$ scores, T-scores and IPAQ scores. Patients were classified as under 50 years, 50-60 years, and over 60 years and comparisons were performed according to these age groups.

\section{Statistical analysis}

The IBM SPSS version 23.0 software (IBM Corp., Armonk, NY, USA) was used for statistical analyses. The Mann-Whitney $U$ test was used for comparison of nonparametric continuous variables. Fisher's exact test and chi-square tests were used for comparison of categorical variables. $\mathrm{P}<0.05$ was considered statistically significant. 


\begin{tabular}{|c|c|c|c|c|c|c|c|c|c|}
\hline \multicolumn{10}{|c|}{$\begin{array}{c}\text { TABLE } 1 \\
\text { Comparison of demographic characteristics of patients }\end{array}$} \\
\hline & \multicolumn{4}{|c|}{ Natural menopause $(\mathrm{n}=220)$} & \multicolumn{4}{|c|}{ Surgical menopause $(\mathrm{n}=65)$} & \multirow[b]{2}{*}{$p$} \\
\hline & $\mathrm{n}$ & $\%$ & Median & Min-Max & $\mathrm{n}$ & $\%$ & Median & Min-Max & \\
\hline Age (year) & & & 57 & $40-78$ & & & 57 & $44-74$ & 0.746 \\
\hline Body mass index $\left(\mathrm{kg} / \mathrm{m}^{2}\right)$ & & & 29.1 & $19.2-49.1$ & & & 29.8 & $22.6-41.2$ & 0.638 \\
\hline Menopause age (year) & & & 48 & $28-60$ & & & 47 & $19-58$ & 0.148 \\
\hline Duration of menopause (year) & & & 9 & $1-37$ & & & 8 & $1-33$ & 0.995 \\
\hline Fracture history & & & & & & & & & 0.792 \\
\hline Yes & 17 & 7.8 & & & 4 & 6.1 & & & \\
\hline No & 203 & 92.2 & & & 61 & 93.8 & & & \\
\hline Fracture in family & & & & & & & & & 0.985 \\
\hline Yes & 17 & 7.8 & & & 5 & 7.7 & & & \\
\hline No & 203 & 92.2 & & & 60 & 92.3 & & & \\
\hline Cigarette use & & & & & & & & & 0.265 \\
\hline Yes & 28 & 12.7 & & & 5 & 7.7 & & & \\
\hline No & 192 & 87.3 & & & 60 & 92.3 & & & \\
\hline Steroids & & & & & & & & & 0.592 \\
\hline Yes & 5 & 2.3 & & & 0 & 0 & & & \\
\hline No & 215 & 97.7 & & & 65 & 100 & & & \\
\hline Previous fracture & & & & & & & & & 0.792 \\
\hline Yes & 203 & 92.3 & & & 61 & 93.8 & & & \\
\hline No & 17 & 7.7 & & & 4 & 6.2 & & & \\
\hline
\end{tabular}

\section{RESULTS}

The surgical menopause group consisted of 65 patients who had bilateral salpingoopherectomy before natural menopause. There was no statistically significant difference between the natural and surgical menopause groups in terms of age, BMI, duration of menopause, smoking, and history of fracture $(\mathrm{p}>0.05)$ (Table 1). There was no difference in terms of the medications used and presence of chronic diseases $(\mathrm{p}>0.05)$.
When the FRAX ${ }^{\circledR}$ scores of the two groups were compared, the risk of major osteoporotic fracture and hip fracture calculated without adding BMD was similar $(\mathrm{p}=0.417$ and $\mathrm{p}=0.234)$. The risk of hip fracture calculated with the addition of BMD was higher in patients with natural menopause $(\mathrm{p}=0.023)$ (Table 2).

Under 50 years of age, femoral neck BMD values were found to be lower in patients with natural menopause compared with patients with surgical menopause $(p=0.021)$. Lumbar vertebrae $\mathrm{T}$-scores were

\begin{tabular}{|c|c|c|c|c|c|}
\hline \multicolumn{6}{|c|}{$\begin{array}{c}\text { TABLE } 2 \\
\text { Comparison of clinical findings }\end{array}$} \\
\hline & \multicolumn{2}{|c|}{ Natural menopause $(n=220)$} & \multicolumn{2}{|c|}{ Surgical menopause $(n=65)$} & \multirow[b]{2}{*}{$p$} \\
\hline & Median & Min-Max & Median & Min-Max & \\
\hline \multicolumn{6}{|c|}{ The 10-year probability of fracture risk without Femur Neck BMD } \\
\hline Major osteoporotic & 3.9 & $2-19$ & 3.6 & $2.5-9.5$ & 0.417 \\
\hline Hip fractures & 0.5 & $0.1-8$ & 0.4 & $0.1-3.1$ & 0.234 \\
\hline \multicolumn{6}{|c|}{ The 10-year probability of fracture risk with Femur Neck BMD } \\
\hline Major osteoporotic & 4.2 & $1-29$ & 3.9 & $2.5-14$ & 0.166 \\
\hline Hip fractures & 0.6 & $0-20$ & 0.3 & $0-5$ & 0.023 \\
\hline \multicolumn{6}{|l|}{ T-Score } \\
\hline Femur neck & -1.25 & $-3.4-+2.9$ & -0.8 & $-3.1-+1.5$ & 0.019 \\
\hline Lumbar & -1.8 & $-3.8-+1.3$ & -1.4 & $-3.3-+0.9$ & 0.052 \\
\hline IPAQ (MET) & 181.5 & $0-10890$ & 165 & $0-1386$ & 0.966 \\
\hline
\end{tabular}




\begin{tabular}{|c|c|c|c|c|c|}
\hline \multicolumn{6}{|c|}{$\begin{array}{c}\text { TABLE } 3 \\
\text { Comparison of bone mineral density and fracture risks in different age groups }\end{array}$} \\
\hline & \multicolumn{2}{|c|}{ Natural menopause } & \multicolumn{2}{|c|}{ Surgical menopause } & \multirow[b]{2}{*}{$p$} \\
\hline & Median & Min-Max & Median & Min-Max & \\
\hline \multicolumn{6}{|c|}{ Femur neck BMD } \\
\hline$<50$ years & -1.1 & $-3.4-1.1$ & -0.1 & $-2.8-1.5$ & 0.021 \\
\hline $50-60$ years & -1.2 & $-2.90-2.90$ & -0.55 & $-2.5-0.9$ & 0.024 \\
\hline$>60$ years & -1.4 & $-3-1.30$ & -1.6 & $-3.1-0.9$ & 0.672 \\
\hline \multicolumn{6}{|l|}{ Lumbar BMD } \\
\hline$<50$ years & -1.7 & $-3.3-2.5$ & -1.2 & $-2.9-0.3$ & 0.077 \\
\hline $50-60$ years & -1.6 & $-3.9-1$ & -1.4 & $-3.3-0.6$ & 0.398 \\
\hline$>60$ years & -2.0 & $-3.8-0.9$ & -2.0 & $-3-0.9$ & 0.330 \\
\hline \multicolumn{6}{|c|}{$\begin{array}{l}\text { The } 10 \text {-year probability of fracture risk without } \\
\text { femur neck BMD (\%) without neck BMD (\%) }\end{array}$} \\
\hline \multicolumn{6}{|c|}{ Major osteoporotic } \\
\hline$<50$ years & 3.5 & $2.6-8.5$ & 3.5 & $2.7-7.2$ & 0.633 \\
\hline $50-60$ years & 3.6 & $2-14$ & 3.5 & $2.5-8.5$ & 0.654 \\
\hline$>60$ years & 5.1 & $2.8-19$ & 4.5 & $3-9.2$ & 0.147 \\
\hline \multicolumn{6}{|l|}{ Hip fractures } \\
\hline$<50$ years & 0.3 & $0.1-1.6$ & 0.2 & $0.1-0.5$ & 0.173 \\
\hline $50-60$ years & 0.4 & $0.1-2.0$ & 0.4 & $0.1-2.0$ & 0.396 \\
\hline$>60$ years & 0.95 & $0.3-8$ & 0.8 & $0.4-3.1$ & 0.165 \\
\hline \multicolumn{6}{|c|}{$\begin{array}{l}\text { The } 10 \text {-year probability of fracture risk with } \\
\text { femur neck BMD (\%) }\end{array}$} \\
\hline \multicolumn{6}{|c|}{ Major osteoporotic } \\
\hline$<50$ years & 4.4 & $3.2-29$ & 3.6 & $3.1-7.9$ & 0.041 \\
\hline $50-60$ years & 4.2 & $2.4-18.0$ & 3.45 & $2.6-14$ & 0.177 \\
\hline$>60$ years & 4.95 & $1-13$ & 5 & $2.5-11$ & 0.784 \\
\hline \multicolumn{6}{|l|}{ Hip fractures } \\
\hline$<50$ years & 0.4 & $0-20$ & 0.1 & $0-4.30$ & 0.028 \\
\hline $50-60$ years & 0.5 & $0-9.3$ & 0.25 & $0-5$ & 0.690 \\
\hline$>60$ years & 0.90 & $0-4.6$ & 0.80 & $0-4$ & 0.672 \\
\hline
\end{tabular}

similar between the two groups $(\mathrm{p}=0.077)$. The median time in menopause in this age group was 3.5 years in the natural menopause group, whereas it was one year in surgical menopause group $(\mathrm{p}=0.027)$. In this age group, major osteoporotic and femoral neck fracture risks calculated with BMD were higher in the natural menopause group $(p=0.041$ and $p=0.028)$. The FRAX ${ }^{\circledR}$ scores calculated without BMD were similar in this age group (Table 3). There was a statistically significant difference in BMD values of the femoral neck between the two groups in patients aged $50-60$ years $(p=0.024)$. However, all clinical findings were similar between the two groups in patients in this age group $(\mathrm{p}>0.05)$.
There was also no difference in FRAX ${ }^{\circledR}$ scores $(\mathrm{p}>0.05)$ (Table 3). Bone mineral density values and FRAX ${ }^{\circledR}$ scores were not different between the two groups after the age of 60 years $(\mathrm{p}>0.05)$ (Table 3$)$.

The physical activity assessment of the patients in the groups was compared using IPAQ, which revealed no difference for IPAQ scores between the two groups.

\section{DISCUSSION}

The FRAX ${ }^{\circledR}$ is an important tool used to determine the 10-year fracture risk in osteoporosis. In our study, FRAX ${ }^{\circledR}$ scores were similar in patients with 
natural and surgical menopause. Bone mineral density measurements did not alter the risk of fracture.

The importance of our study is that it evaluates the risk of fracture due to surgical and natural menopause using $\mathrm{FRAX}^{\circledR}$, and examines the change of FRAX ${ }^{\circledast}$ scores with the addition of BMD measurements in these patients. Although there are studies evaluating the risk of osteoporosis-related fractures in the literature, the number of studies comparing FRAX ${ }^{\circledR}$ scores in these two groups is limited.

There are conflicting results in the literature comparing the BMD of patients with surgical menopause and those with natural menopause. In one study, the mean BMD values were significantly lower in the surgical menopause group compared with the natural menopause group. ${ }^{[8]}$ In a metaanalysis of Fakkert et al., ${ }^{[9]}$ no difference was found between these two groups. In a study investigating the effect of age and menopause type on BMD, the values of patients with surgical menopause and natural menopause were shown to vary in different age groups. Femoral neck T-scores were similar in two groups aged 45-50 and 50-55 years, whereas BMD was higher in patients older than 55 years who underwent surgical menopause. Between the ages of 45-50 years, lumbar vertebrae T-scores were found to be lower in the surgical menopause group compared with the natural menopause group. ${ }^{[10]}$ In our study, lumbar vertebrae $\mathrm{T}$-scores were similar between the two groups regardless of age; we found that femoral neck T-scores were higher in surgical menopause group $(-0.8 v s$. -1.25$)$. In the literature, it is stated that the difference between $\mathrm{T}$-scores in patients with surgical and natural menopause decreases and disappears as the duration of post-menopausal period increases. ${ }^{[9]}$ This may be due to the fact that natural menopause leads to bone loss during the severalyear premenopausal period. This effect disappears more quickly in trabecular bone, but the difference in the femoral neck is maintained for a longer time. In our study, we found that femoral neck T-scores were different between the two groups aged under 60 years, whereas this difference disappeared in patients older than 60 years.

The effect of early rapid bone loss after oophorectomy decreases with a prolonged menopause duration, and BMD becomes similar in the two groups. In our study, the duration of menopause was longer in the natural menopause group in patients younger than 50 years. In this age group, femoral neck BMD values of patients with natural menopause were lower than in those with surgical menopause, and FRAX ${ }^{\circledR}$ femoral neck fracture scores calculated by adding BMD were higher in patients with natural menopause. In our study, we found that this effect disappeared after the age of 50 years with the prolongation of menopause.

It has been shown that bone loss is accelerated in patients undergoing oophorectomy at an early age before natural menopause, but that oophorectomy after natural menopause does not affect bone density. ${ }^{[11]}$ What is important here is the time passed in menopause. ${ }^{[12]}$ In a recent meta-analysis, no significant difference was reported between surgical menopause and natural menopause in terms of BMD and fracture prevalence. ${ }^{[9]}$ This is similar to the results found in our study.

The FRAX ${ }^{\circledast}$ has been one of the most commonly used methods in fracture risk calculation since 2008. When DXA is not available, FRAX ${ }^{\circledast}$ results calculated without BMD can be used to assess fracture risk. ${ }^{[5]}$ In our study, we found that adding BMD results to FRAX $^{\circledR}$ did not affect fracture risk. Therefore, we think that FRAX ${ }^{\circledR}$ scores without BMD can be used to assess fracture risk when BMD measurements cannot be performed. However, it is recommended that in patients with clinical risk factors (e.g. low BMI, glucocorticoid therapy, smoking and alcohol use, immobilization), BMD measurements should be used for FRAX ${ }^{\circledR}$ fracture risk assessment if possible. ${ }^{[5]}$

The lack of physical activity is a well-known risk factor for osteoporosis. ${ }^{[13]}$ The IPAQ was developed in 1998 to conduct global physical activity surveillance. It has been the most widely used physical activity survey ever since. The IPAQ-short form is a sevenitem questionnaire that evaluates activity levels (low, moderate, high physical activity) in the last seven days. ${ }^{[14]}$ In our study, IPAQ scores were very low in most patients. Although there are no studies showing the effect of IPAQ scores on FRAX $^{\circledR}$ in the literature, there are many studies showing the relationship between physical activity and BMD. ${ }^{[15,16]}$ In these studies, BMD values were found to be better in patients with high IPAQ scores. However, in our study, it was not possible to make an interpretation because IPAQ scores of patients with both natural and surgical menopause were quite low.

The limitations of our study can be listed as follows: Although it is a prospective cross-sectional study, the number of patients is limited due to being performed in a single center. In addition to this, consistent with the literature, there was a smaller 
number of patients in the entire patient group who underwent surgical menopause. Fracture risk was compared with FRAX ${ }^{\circledR}$ scores between two groups. However, 10-year follow-up was not performed and actual fracture rates could not be compared. In their study, Melton et al. ${ }^{[17]}$ examined 340 females who had bilateral oophorectomy and reported that the risk of osteoporotic fracture increased after 16 years of median follow-up. The median menopause duration of the study groups had a wide standard deviation. However, in our study, we divided the patients into different age categories and performed subgroup comparisons. There is a need for further studies, in which patients are categorized at shorter intervals according to their menopause duration. With long-term follow-up, the risk of fractures in these age categories can be more clearly revealed.

In conclusion, our study is important since it compares the risk of fracture in patients with natural and surgical menopause using FRAX ${ }^{\circledR}$ scores. Surgical menopause is the result of surgical interventions to treat patients. In these patients, the severity of osteoporosis and the risk of fracture seem not to increase compared to patients who have had natural menopause. Nevertheless, further large-scale and longterms studies are needed to compare the results of surgical and natural menopause.

Declaration of conflicting interests

The authors declared no conflicts of interest with respect to the authorship and/or publication of this article.

\section{Funding}

The authors received no financial support for the research and/or authorship of this article.

\section{REFERENCES}

1. Rodriguez M, Shoupe D. Surgical Menopause. Endocrinol Metab Clin North Am 2015;44:531-42.

2. Parker WH. Ovarian conservation versus bilateral oophorectomy at the time of hysterectomy for benign disease. Menopause 2014;21:192-4.

3. Kanis JA, Harvey NC, Johansson H, Odén A, Leslie WD, McCloskey EV. FRAX Update. J Clin Densitom 2017;20:360-7.
4. Imerci A, Aydogan NH, Tosun K. Agreement between the Turkey Guidelines and the Fracture Risk Assessment Tool ${ }^{\circledR}$ based Intervention Threshold. J Bone Metab 2018;25:79-86.

5. Kanis JA, Cooper C, Rizzoli R, Reginster JY; Scientific Advisory Board of the European Society for Clinical and Economic Aspects of Osteoporosis (ESCEO) and the Committees of Scientific Advisors and National Societies of the International Osteoporosis Foundation (IOF). European guidance for the diagnosis and management of osteoporosis in postmenopausal women. Osteoporos Int 2019;30:3-44.

6. Available at: https://www.sheffield.ac.uk/FRAX/tool. aspx? country=6. [August 07, 2019]

7. Saglam M, Arikan H, Savci S, Inal-Ince D, Bosnak-Guclu $\mathrm{M}$, Karabulut E, et al. International physical activity questionnaire: reliability and validity of the Turkish version. Percept Mot Skills 2010;111:278-84.

8. Chittacharoen A, Theppisai U, Sirisriro R. Bone mineral density in natural and surgically-induced menopause. Int J Gynaecol Obstet 1999;66:193-4.

9. Fakkert IE, Teixeira N, Abma EM, Slart R, Mourits M, de Bock GH. Bone mineral density and fractures after surgical menopause: systematic review and meta-analysis. BJOG 2017;124:1525-35.

10. Hadjidakis DJ, Kokkinakis EP, Sfakianakis ME, Raptis SA. Bone density patterns after normal and premature menopause. Maturitas 2003;44:279-86.

11. Hayirlioglu A, Gökaslan H, Andaç N. The effect of bilateral oophorectomy on bone mineral density. Rheumatol Int 2006;26:1073-7.

12. Kritz-Silverstein D, von Mühlen DG, Barrett-Connor E. Hysterectomy and oophorectomy are unrelated to bone loss in older women. Maturitas 2004;47:61-9.

13. Rittweger J. Can exercise prevent osteoporosis? J Musculoskelet Neuronal Interact 2006;6:162-6.

14. Lee PH, Macfarlane DJ, Lam TH, Stewart SM. Validity of the International Physical Activity Questionnaire Short Form (IPAQ-SF): a systematic review. Int J Behav Nutr Phys Act 2011;8:115.

15. Hartley S, Garland S, Young E, Bennell KL, Tay I, Gorelik A, et al. A Comparison of Self-Reported and Objective Physical Activity Measures in Young Australian Women. JMIR Public Health Surveill 2015;1:e14.

16. Ilesanmi-Oyelere BL, Roy NC, Coad J, Kruger MC. Associations between Self-Reported Physical Activity, Heel Ultrasound Parameters and Bone Health Measures in Post-Menopausal Women. Int J Environ Res Public Health 2019;16:3177.

17. Melton LJ 3rd, Khosla S, Malkasian GD, Achenbach SJ, Oberg AL, Riggs BL. Fracture risk after bilateral oophorectomy in elderly women. J Bone Miner Res 2003;18:900-5. 